\title{
Meteorological Forecasting for renewable energy plants. A case study of two energy plants in Spain
}

\author{
Andrés Robalino-López ${ }^{1}$, Ángel Mena-Nieto ${ }^{2}$ \\ ${ }^{1}$ Escuela Politécnica Nacional (EPN) - Ecuador, andres.robalino@epn.edu.ec, \\ ${ }^{2}$ Universidad de Huelva (UHU) - Spain,mena@uhu.es.
}

\begin{abstract}
Energy resources are the engines that drive every economy $[1,2,3]$, Therefore, it is necessary to develop their exploitation in a friendlier, environmentally and sustainable way indeed it is a critically needed nowadays. Then, it is necessary to improve efficiency and optimize renewable energy in order that replace polluting energy sources. This work aims to relate the use of forecasting on meteorological variables such as wind speed, wind direction, solar radiation, among others, obtained by mathematical models implemented on computer to forecast energy production in renewable energies plants. It has been implemented and automated one of the most used models by the scientific community in this field, WRF (Weather Research and Forecasting Model). WRF is a next generation mesoscale model, designed to serve as a tool for meteorological research in addition to provide forecasts in operational regime. This research introduce the topic of energy forecast, mainly of renewable energy, focusing on wind and solar energy, basing the study on a better forecasting of meteorological variables in order to use as income in energy production forecast. A case study in two Spanish renewable energy plants is exposed.
\end{abstract}

Keywords: Energy forecasting, meteorological model, WRF.

\section{INTRODUCTION}

The state of the atmosphere is always a conditioning factor of human activity. Weather forecasting is a staple tool for today's society [4,5]. Moreover, the complexity of the physical processes involved imply the need for intensive calculations using numerical models for its resolution, with a high computational cost and sometimes also with human cost. In addition, the non-linear nature of the involved processes limits the temporal validity of the forecasts made, so these need to be recalculated, based on new experimental data $[6,7]$.

The physics of the atmosphere tries to understand and explain the thermodynamic and transport processes involved in the evolution of the atmosphere in a deterministic manner. The equations of energy conservation and continuity are established as coupled systems through differential equations that describe the atmospheric dynamics. In general, this system of differential equations does not allow an analytical solution, so that's why the use of techniques for solving numerical calculations is necessary. The validity of the results obtained through simulation depends greatly on the quality of the physical model used, accuracy of numerical methods implemented for resolution and of data fed into its models.

Deterministic approach used in the description of atmospheric dynamics allows making forecasts of conditions from different variables of interest from the initial conditions [8]. However, the nonlinear nature of the equations governing the evolution of the system implies a certain chaotic nature which affects the validity of the long-term forecast $[9,10]$.

In this context a meteorological model is a set of differential equations (involving the majority of atmospheric physics variables) whose complexity resolution is translated into a computer program that produces meteorological information to a time in the future for certain parts of the world and certain altitudes $[1,12]$.

The models are based on data from meteorological probe, weather satellites, and ground observations. These observations are processed by data assimilation and objective analysis methods that perform quality control and obtain values used by mathematical algorithms [13]. Then these data are used in the model as a starting point for forecasting.

The calculations performed by these equations begin to use meteorological data and determine the rates of change of different atmospheric variables. The rates of change forecast the state of the atmosphere within a short time in the future. The following equations apply to this new state of atmosphere to calculate new rates of change, and those new rates forecast a state of atmosphere for a next time in future $[14,15]$. This process of small increments over the 
time is repeated continuously until the solution reaches time wanted, to obtain the forecast. The time lapse of each temporary increase depends on the distance at which two points are on the grid, or spreadsheet cell. Time steps in global climate models may be on order of ten minutes, while time steps used in regional models may vary from a few seconds to a few minutes [16].

The MM5 (Mesoscale Meteorological Model 5th generation) model is well known within the international scientific community, which has been validated in a number of works $[17,18]$. Currently there is an advanced version of that model known as WRF (Weather Research and Forecasting Model). WRF Mesoscale Model is a next generation, designed to serve as a tool for atmospheric researches in addition to provide forecasts in operational regime [3,24].

On the other hand, energy resources are the engines that drive every economy $[2,3]$, the need to develop their exploitation in an environmentally friendlier and sustainable manner is a critically needed nowadays. Due to that, it is necessary to improve their efficiency and optimize it; in order to finally they can replace the polluting energy sources.

This research sought to introduce the topic of energy forecasting, mainly from renewable energy sources, focusing on wind and photovoltaic. Basing the study on improving the input data (meteorological variables) for energy forecast models in these kinds of energy plants.

To obtain a better forecast of weather variables (irradiation, wind speed and direction, etc.) is essential to improve the forecast system reliability in these plants [11,21]. This work is focused on the implementation and automation of a numerical weather forecasting models under a computer system. Particular goals of this research were the implementation and automation ${ }^{1}$ of a numerical weather forecast model (WRF) for automatic daily forecasting in two domains (Iberian Peninsula and Northern Spain) with spatial resolutions of 27 and 9 $\mathrm{km}$, respectively, in order to improve forecasting process in the production of energy in two selected renewable energy plants.

The paper are divided in four sections, the first one shows the introduction about the topic under study. The second introduces the materials and methods used in this research. In the third section are presented two studies cases where was implemented the energy forecast on base of the forecast of meteorology variables. Finally the conclusions are presented.

\footnotetext{
${ }^{1}$ Computer Automation: the use of computerized systems or electromechanical elements for controlling machinery and/or process replaces human operators. In the context of this paper refers to the creation of software for autonomous execution of the meteorological model.
}

\section{MATERIALS AND METHODS}

\section{A. Meteorological model WRF}

The structure of manuscripts:

WRF is an Eulerian ${ }^{2}$, non-hydrostatic ${ }^{3}$ and compressible ${ }^{4}$ model. Their vertical coordinates are from hydrostatic pressure and the use of an Arakawa ${ }^{5}$ C-grid. WRF is suitable for a wide spectrum of applications across scales, ranging from meters to thousands of kilometers. Fig. 1 shows the basic flow of WRF model execution.

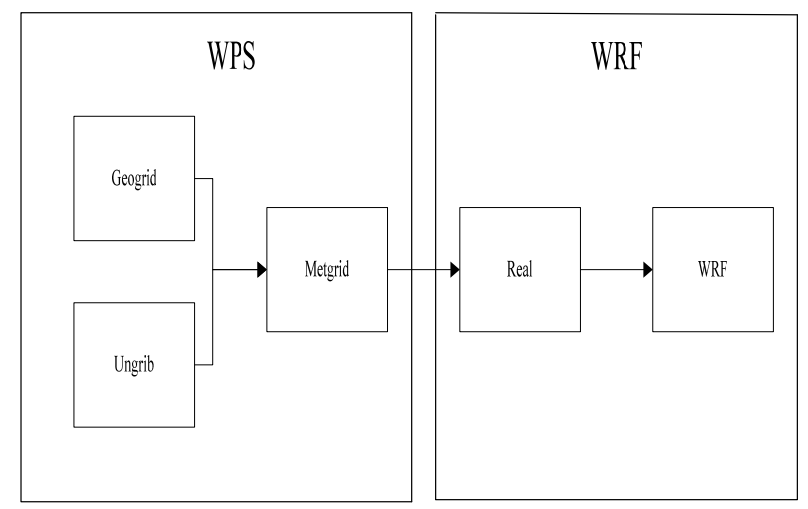

Fig. 1. Basic flow of WRF model execution (Michalakes et al., 2004)

WRF Preprocessing System (WPS) is the module responsible for performing the pre-processing of information. This module consists of the following components: i) geogrid.exe that interpolates the terrestrial data for calculation domains (Static), ii) ungrib.exe that unpack the GRIB weather data and pack it into intermediate format files, iii) metgrid.exe that interpolate horizontal meteorological data within the domain of the model.

WRF is the main module of the model and is responsible for conducting the simulation, it consists of: i) real.exe that interpolates vertically the met_em files (generated metgrid.exe), creating boundaries and initial conditions files and some evidence of consistency, ii) wrf.exe that generates the model forecast.

\footnotetext{
${ }^{2}$ Eulerian model: a fixed reference system (usually the focus issuer) is established, and tries to solve the balance equations of mass, energy and momentum, with different degrees of approximation.

${ }^{3}$ No hydrostatic model: includes a predictive equation for the vertical movements. This allows direct processes include hydrostatic or buoyancy and dynamic pressure perturbations. In contrast, hydrostatic models have no prognostic equations for vertical movements and can only indirectly include the effects of buoyancy.

${ }^{4}$ Compressible: compressibility of flow is basically a measure of the change in density. The gases are generally very compressible; however, most liquids have compressibility.

${ }^{5}$ Arakawa: This grid system shows different ways to represent and calculate physical orthogonal quantities, in particular speed and the masses of the corresponding quantities.
} 


\section{B. Type of input and output data}

The input data required for the implementation of WRF both prediction mode, and re-analysis mode, come from the Global Forecasting System (GFS) that is a global forecast model by NOAA [22,23]. The reason for using WRF in this work is to increase the resolution and accuracy of the forecast on the domains under the study.

The GFS model runs four times per day and produces forecasts up to 16 days in advance, but with decreasing spatial and temporal resolution. It is widely agreed that forecast of more than 7 days is very general and is not accurate enough. The model is executed in two parts: the first has a higher resolution; it goes up to 180 hours (7 days) in the future, the second part goes from 180 to 384 hours (16 days) on a lower resolution. The resolution varies in each part of the model: Horizontal, splits the surface of the earth cells in 35 or $70 \mathrm{~km}$. Vertical, divides the atmosphere into 64 layers and through time produces a forecast for every 3 hours for the first 180 hours, after which are produced for every 12 hours.

Input files for WRF have a temporal spatial resolution of 35-70 $\mathrm{km}$ and 3 or 6 hours depending on whether are forecasting or re-analysis. The format of these files is GRIB2. GRIB (gridded binary) is a mathematically concise data format, commonly used in meteorology to store historical and forecast meteorological data. As output, WRF returns a NetCDF files (Network Common Data Format). The feature of this format is that it contains enough information to know what kind of data is in the file (variable, units, dimensions, institution that created it, etc.), unlike other formats that require an additional file for proper interpretation.

Data analysis and generation of the figures and maps were carried out by NCAR Command Language (NCL) routines. This is an interpreted language designed specifically for analysis and visualization of scientific data.

\section{Execution Model Formats}

This research raised two formats of model configuration: a) Forecasting for 48 hours. In this configuration the inputs to the model are from the first forecast of the GFS $(0: 00 \mathrm{H})$ with a forecast up to 48 hours on the domains under the study. The speciations of the files used for forecasting process are i) Name: gfs.t00z.pgrbf00.grib2. ii) Model: GFS. Iii) Cycle: 00Z. iv) Forecast: analysis. v) Number of Records: 299. b) Reanalysis of 48 hours. In this configuration the input data also come from one of the executions of the GFS, but data that is used in this case, contain information from data assimilation modules and a greater number of observations. The output data of the assimilation process (National Centers for Environmental Prediction, NCEP FNL) have a resolution of $1.0 \times 1.0$ degrees and are operationally prepared every six hours. These come from the system of global data assimilation (GDAS) that is continuously collecting data from observation of the Global Telecommunications System (GTS) and other sources, which are subjected to many tests for interpretation and final assimilation. Therefore, with a greater amount and more reliable data as input for WRF a reanalysis simulation of the last 48 hours is obtained as result of this configuration.

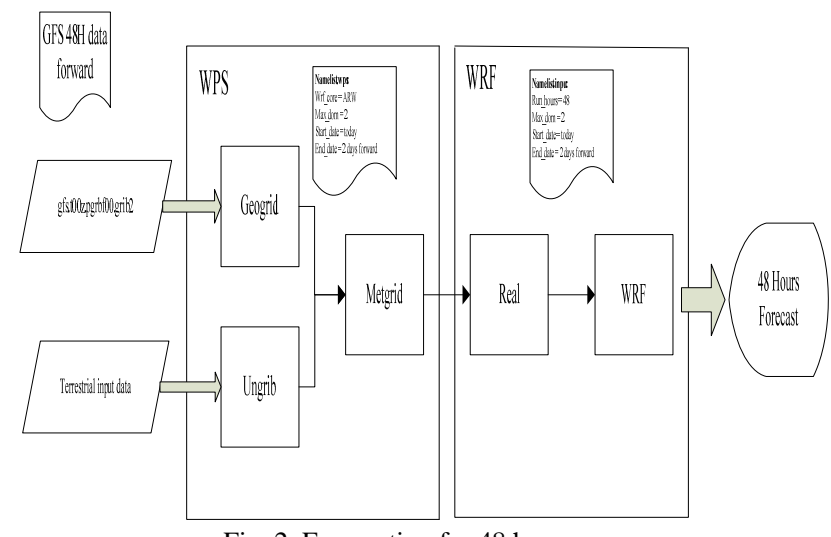

Fig. 2. Forecasting for 48 hours



Fig. 3. Reanalysis for 48 hours

\section{Operating Mode}

The operational mode refers to the formats of operational configuration of the model for specific purposes of this research. To create these modes was used the formats discussed above, implementation tailored to each of demands. In this case the mode runs a 48-hour forecasting simulation for the next two days and five 48-hours reanalysis simulations - for the five days immediately preceding execution date. This configuration is achieved to obtain a weather forecast (methodological variables) for the next 48 hours over selected domains in less than 20 minutes.

Moreover, reanalysis data (Simulations with GDAS) for the last 5 days help to conduct validation studies and test the meteorological model, and energy forecasters as well. 


\section{E. Operating Mode}



Fig. 4. Model automation scheme

The Fig. 4 shows the general operation flow chart of the automation program. The different execution activities of the model were divided in order to achieve the automation. These activities were programmed into independent modules that execute defined tasks to be linked into a global structure (Allrun_xx.sh script file). In this way the overall process is performed by executing a single script thread launching necessary to achieve the task. The automation was performed by including the general script in the scheduled list of the system (cron daemon) under Linux.

Further is described precisely the purpose of each of the modules, in addition - the corresponding scripts and main characteristics also are mentioned: i) Cleanup Module (M1 Cleaning): The overall purpose of this module is to delete all additional files that have been created in the last run of the model. It has a single script for forecasting and reanalysis modes. The module routines run in less than one second. ii) Data Collection Module (M2 Get Data): Daily, this module downloads all data GRIB2 which is necessary for the model to start to work. The forecast modes have a unique script and the reanalysis modes uses three files that perform and set the download field, do the downloading and ordering of the input files respectively. These routines run in an average of five minutes, depending on the availability of data on the remote server. iii) Module creation initialization file (M3 Make Name List): The module aims is to create initialization files (namelist.wps and namelist.input), which are used by the model to start the simulation. This module has two autonomous scripts in order to generate both files in each configuration. The first file determines simulation hours, start date, end date, time interval of the input files, georeferencing and resolving domains for the WPS module. The second file determines simulation hours, start and end dates, georeferencing and physical models used in the simulation to run the WRF module.

The average execution of this module is from 1 to 5 seconds, depending on execution format and it is an independent module of the input data. iv) WPS preprocessing module (M4 WPS): The module is responsible for automating task of the WPS preprocessing. The module performs the automatic execution of all necessary subroutines to run WPS. This module is dependent on the input data and 
previous made for previous modules and it has four scripts: EXECUTED link_grib (1 second), EXECUTED geogrid (25 seconds), EXECUTED ungrib (60 seconds), EXECUTED metgrid (120 seconds) MODULE EXECUTED WPS (206 seconds). v) WRF simulation module (M5 WRF): This module is responsible for automating WRF simulation tasks. Module performs automatic execution with all necessary simulation subroutines to run WRF. This module is dependent on the generated data in the preprocessing WPS module and in the generating initialization files process. It is the module that demands more computing and time resources. It has four scripts: EXECUTED copy meth (5 seconds), EXECUTED Real (25 seconds), EXECUTED wrf (12 minutes), EXECUTED copy out (60 seconds). vi) Module automatic validation (M6 Validation): The purpose of this module is to provide an automatic validation of the reliability of the model used for weather forecasting. It is accomplished by comparing output data of the forecasting processes with the output data of the reanalysis process for each of the variables analyzed. It has 1 script that has an average 60 seconds execution time.

\section{F. Validation Format-Meteorological Model}

The validation process of the output data of weather model has been set using a routine that calculate the mean square deviation (RMSD) with the forecasting and re-analysis data for each simulation period and each spatial point of the domain under study. In this way an efficient evaluation of effectiveness of the forecast is achieved continuously. The RMSD is widely used in a quantizer verification of numerical models and is defined as:

$$
\operatorname{RMSD}\left(\theta_{1}-\theta_{2}\right)=\sqrt{E\left(\left(\theta_{1}-\theta_{2}\right)^{2}\right)}=\sqrt{\frac{\left.\sum_{i=1}^{n}\left(x_{1, i}-x_{2, i}\right)\right)^{2}}{n}}
$$

Where $\theta_{1}$ represents data generate in the forecasting process, $\theta_{2}$ represents data generate in the process of re-analysis and at the same time $\theta_{1}$ y $\theta_{2}$ are defined as:

$$
\theta_{1}=\left[\begin{array}{c} 
\\
x_{1,1} \\
x_{1,2} \\
\cdot \\
x_{1, n}
\end{array}\right] \quad \theta_{2}=\left[\begin{array}{c} 
\\
x_{2,1} \\
x_{2,2} \\
\cdot \\
x_{2, n}
\end{array}\right]
$$

$n$ is the number of points used for the forecasting and reanalysis process.

Specifically, the RMSD process takes a point within the forecast domain and subtracting it from its counterpart in the re-analysis domain to find the specific deviation, then it has to be squared to remove negative factors and a sum is performed within the entire interval $n$, it is divided to the number of samples to get the average value; finally the square root is removed. This procedure extends it to all points within the timeline by which was made the forecast.

Fig. 5 shows the RMSD time trends of two wind components ( $\mathrm{V}$ and $\mathrm{U}$ ) for 48-hours simulation. In general, the RMSD follows the same trend and has the same magnitude for all domains under the study and for every period of 48 hours. RMSD value is increasing as the forecasting period increases. This is due to accumulation of errors in both the forecast and re-analysis process, besides the non-linearity of the differential equations that govern these physical phenomena.
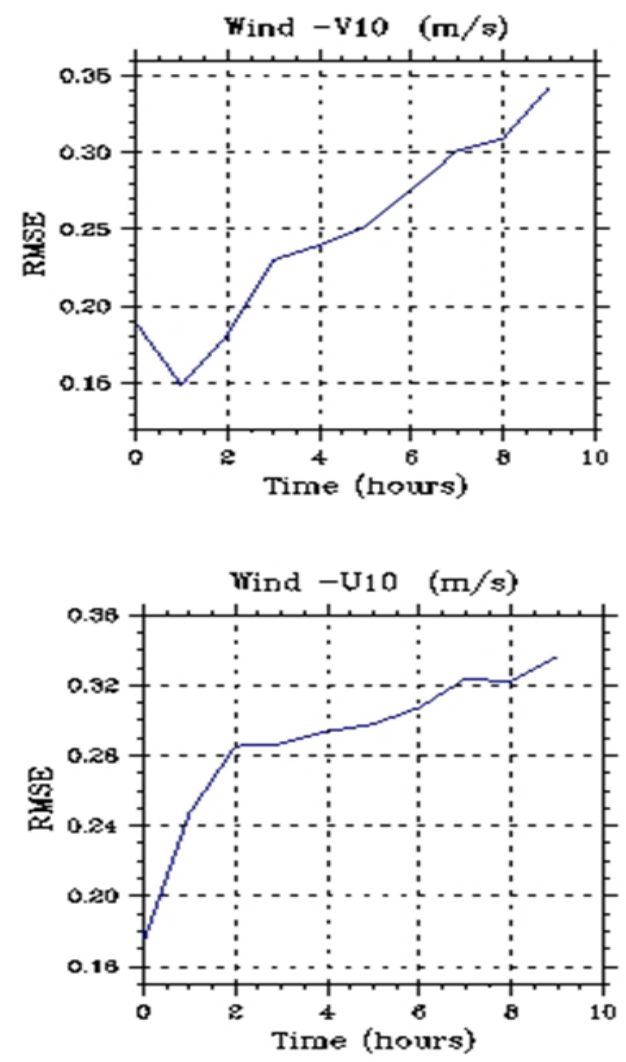

Fig. 5. Validation (RMSD between forecasting and data re-analysis data for domain 1 for variables $\mathrm{U}$ and $\mathrm{V}$ wind).

\section{CASES STUDY}

\section{A. Case study: Wind energy plant (Cabo Vilan, Spain)}

In this section is presented a case study of a wind energy plant in the north of Spain, for which is taken real data from a meteorological stations controlled by AEMET (Agencia Estatal de Meteorología, Spain), which had been contrasted with the data obtained by the execution of the model in order to forecast the energy production in a wind turbine in the plant. The period of analysis is 61 days. Data recorded by the meteorological station at Cabo Vilan, in the province of A Coruña, Spain, from the network of stations AEMET, the frequency of the data available were 10 
minutes. The geo-position is: Latitude: 43.160556, Longitude: -9.210833, Altitude: 50 meters.

The Fig. 6 shows the average wind speed, it can be observed that both modeled and experimental data show similar patterns, particularly in qualitative aspects (in the manner that they shown variations). On the quantitative aspect, we can see less similarity, where it can be seen that the curve from the model is softer than from experimental data pattern.



Fig. 6. Average wind speed. Blue curve represents data from the model and red curve represents experimental data.

An important feature of wind energy is that the power output of a wind turbine is proportional to the cube of the wind speed (Hellmann's exponential law) and a own characteristic curve-potential of each turbine. Therefore a greater precision is required in the data associated with this type of action than for other applications. In this study were used the potentialcurve that shows it on Fig. 7 (theoretical curve), it represents standard conditions: Ambient $15 \circ \mathrm{C}$, pressure 1013 mbar and air density of $1.225 \mathrm{~kg} / \mathrm{m} 3$, rotor blades clean and no disturbed horizontal airflow.

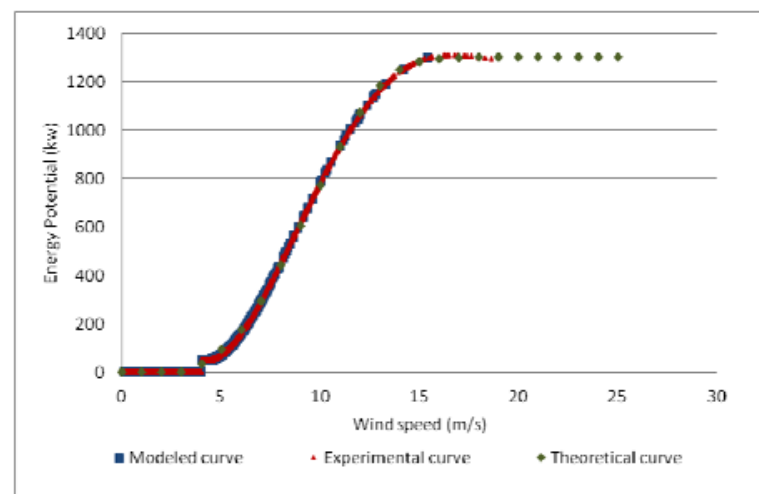

Fig. 7: Potential-curve. Blue curve represents modeled curve, red represents experimental curve and green represents theoretical curve.



Fig. 8. Energy production from a wind turbine. Blue curve represents data from the model and red curve represents experimental data. 
Now, in order to get energy forecast, was used the data set from a model on this specific variable and the potential-curve presented above.

Fig. 8 shows both curves, first that come from the model and the second comes from experimental data; we can discern that there is some similarity between two curves, especially in qualitative terms. However in quantitative aspect there is already a major difference caused mainly, as is mentioned before, by the accumulation of errors of the average wind speed variable that come from meteorological model. The mean square deviation for each of considered variables (wind speed and energy production) was performed. There are some values that represent major errors, therefore they should be discarded in order to neither distort nor hinder the study. Regardless of these major errors, which are specific values, most points considered, show a standard deviation about 3.5 for wind speed variable and 352 for energy production. Once again, it should be mentioned that the resulting error in the energy production is proportional to the cube of error introduced in the average wind speed of the meteorological model because of the cubic relationship between these two, in our specific study case this proportion was around four times. On the other hand the total forecasted energy production by the model during the studied period was around $124 \mathrm{Mw}$ meanwhile the experimental energy production was around $339 \mathrm{Mw}$ by turbine (63.5\% of error).

\section{B. Case Study: Photovoltaic energy plant (Valladolid, Spain)}

In this section is presented a case study of a photovoltaic energy plant in the north of Spain, for which are taken real data from the photovoltaic plant of Valladolid, which had been contrasted within obtained data by running model and treated to forecast the energy production. The period of analysis is 61 days. The geo-position is: Latitude: 41.6451, Longitude: -4.8146.

Fig. 9 shows the irradiation variable, as in the wind case it can be observed that both modeled and experimental data show similar patterns, particularly in qualitative aspects (in the manner that they shown variations). On the quantitative aspect, we can see less similarity, where it can be seen that the curve from the model is softer than from experimental data pattern, but certainly shows a better approximation than wind case.

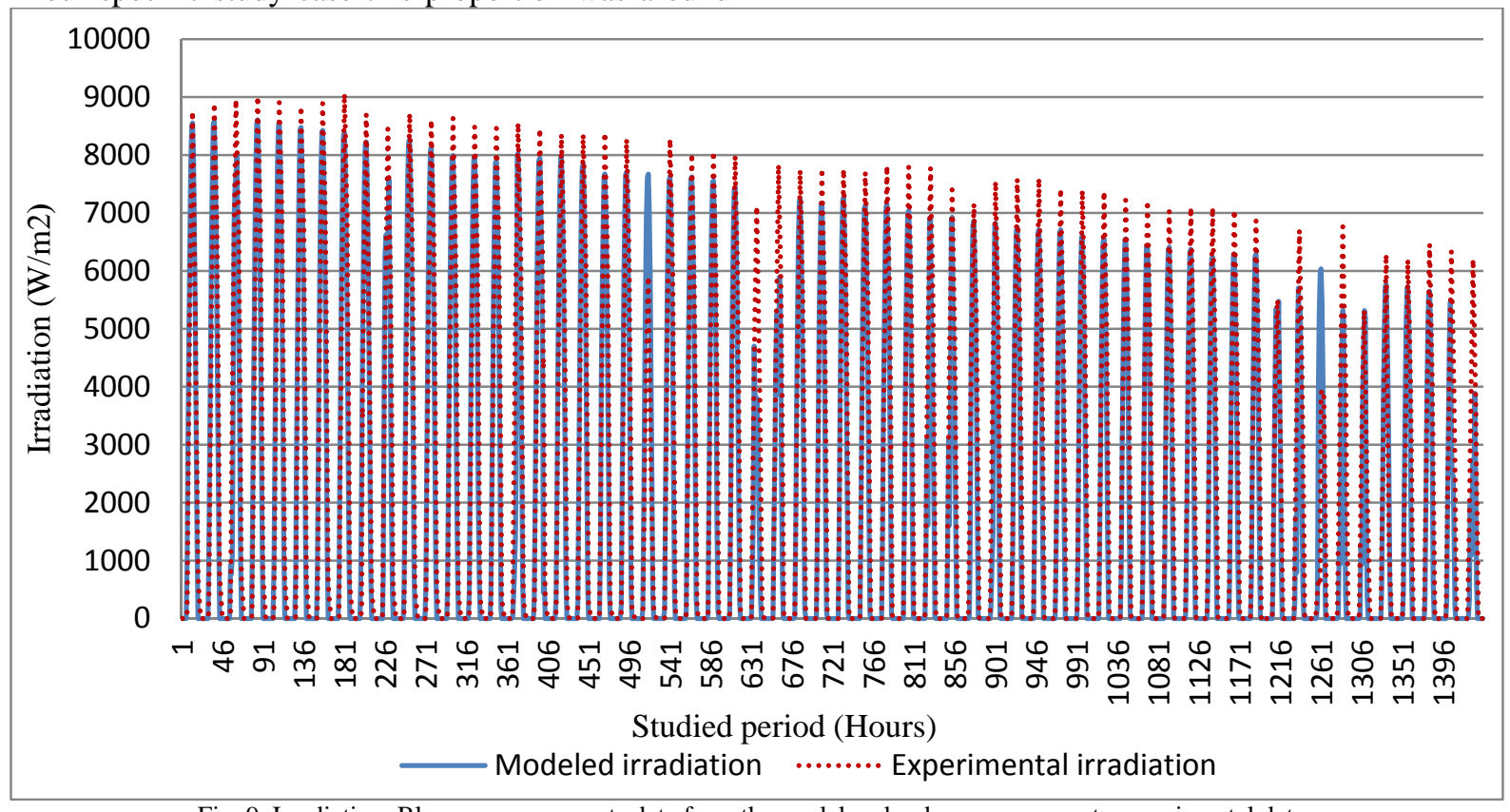

Fig. 9. Irradiation. Blue curve represents data from the model and red curve represents experimental data.

In order to forecast energy productions, in this case have been energy forecasters, which take as input variable the solar radiation (meteorological variable). This forecaster was constructed, based on experimental data of production from photovoltaic plant as an intervals function, like it is shown in Formula 4. Note that, the resulting forecast error is dependent of the forecaster used.

$$
\operatorname{Eng} P= \begin{cases}0 & \text { if radiation }<1860 \\ 8654,8^{*} \operatorname{Ln}(\text { radiation })-63011 & \text { if radiation } \geq 1860\end{cases}
$$

As in wind case, we can see that there is a similarity between these two, especially in the qualitative aspect, regarding to the actual energy production data. However in the quantitative aspect there is already a major error, caused primarily, as mentioned before, by the accumulation of errors from the meteorological forecast model and from the energy forecaster used. Also, the mean square deviation for each of considered variables (irradiation and energy prqduction) was performed. Therefore most points considered show a standard deviation about 895 for 
irradiation variable and 3782 - for energy production. On the other hand the total forecasted energy production by the model during the studied period was

around $6025 \mathrm{Mw}$ meanwhile the experimental energy production was around $5392 \mathrm{Mw}(10.5 \%$ of error).



Fig. 10. Energy production from the photovoltaic plant. Blue curve represents data from the model with static forecaster and red curve represent experimental data.

\section{CONCLUSIONS}

In order to have an optimal manage of renewable energy sources such as wind or solar, it is essential to have a system of accurate, robust and reliable energy forecast. Better understanding and accuracy in the forecasting of meteorological variables affect directly the forecasting of energy in plants that use the natural resources mentioned. An accurate forecast can generate significant both economic benefits and energy efficiency, especially in electricity markets where there are penalties by inaccurate energy forecasts (liberalized markets). On the other hand, In these markets also there are benefits when the forecasts are conform to the actual energy production. So, to ensure greater profitability to renewable energy plants, it is essential that energy producers have a reliable energy forecast system.

Case studies showed significant deviations in the forecast of the variables under the study. However it is important to mention that further improvement in energetic forecasters would significantly improve the results obtained with these systems. This improvement in these forecasters has been outside of the scope of the study but is a line of research to continue. Also it is important to mention that the variations, observed in cases study, are due to some specific circumstances, such as: intrinsic special characteristics of the study site (land mass, front water mass), the accumulation of errors in the model and non-linearity of differential equations governing the phenomenon.

\section{$\mathrm{V}$ REFERENCES}

[1] A. Robalino-López, J.E. García-Ramos, Antonio A. Golpe, Á. Mena-Nieto. System dynamics modelling and the environmental Kuznets curve in Ecuador (1980-2025). Energy Policy 67 (2014) 923-931, 2014.

[2] C. Chan. The State of the Art of Electric, Hybrid, and Fuel Cell Vehicles. Proceedings of the IEEE. Vol.95,No.4, 2007.

[3] M.T. Brown, S. Ulgiati. Energy quality, emergy, and transformity: H.T. Odum's contributions to quantifying and understanding systems. Ecological Modelling. Vol. 178, Iss 1-2, 2004.

[4] J. Verdin, C. Frunk, G. Senay, Choularton R. Climate science and famine early warning. Philosophical transactions of the Royal Society. Vol. 360, Iss. 2155, October 2005.

[5] V. Coates, M. Farooque, R. Klavans, K. Lapid, H. A. Linstone, C. Pistorius, A. L. Porter. On the Future of Technological Forecasting. Technological Forecasting and Social Change. Vol. 67, Iss. 1, 2001.

[6] K. Beiven. Towards an alternative blueprint for a physically based digitally simulated hydrologic response modelling system. Hydrological Processes.Vol. 16, Iss. 2, 2002.

[7] P. Young. Data-based mechanistic modelling of environmental, ecological, economic and engineering systems. Environmental Modelling \& Software. Vol. 13, Iss. 2, 1998.

[8] T. N. Palmer. A nonlinear dynamical perspective on model error: A proposal for non-local stochastic-dynamic parametrization in weather and climate prediction model. Quarterly Journal of the Royal Meteorological Society. Vol. 127, Iss. 572, 2001.

[9] M.N Islam, B Sivakumar. Characterization and prediction of runoff dynamics: a nonlinear dynamical view. Advances in Water Resources. Vol. 25, Iss. 2, 2002.

[10] Tessaleno C. Devezasa, , James T. Corredine. The nonlinear dynamics of technoeconomic systems: An informational interpretation. Technological Forecasting and Social Change. Vol. 69, Iss. 4, 2002

[11] F. X. Le Dimet, O. Tailagrand. Variational algorithms for analysis and assimilation of meteorological observations: theoretical aspects. Tellus. Vol. 38A Iss. 2, 1986. 
[12] O. Talagrand,, P. Courtier. Variational Assimilation of Meteorological Observations with the Adjoint Vorticity Equation. I: Theory. Quarterly Journal of the Royal Meteorological Society. Vol. 133, Iss. 478, 1987.

[13] P. L. Houtekamer, H. L. Mitchell. A Sequential Ensemble Kalman Filter for Atmospheric Data Assimilation. Monthly Weather Review. Vol.129, Iss. 1, 2001.

[14] D. B. Botkin, H. Saxe, M. B. Araújo, R. Betts, R. H. W. Bradshaw, T. Cedhagen, P. Chesson, T. P. Dawson, J. R. Etterson, D.l P. Faith, S. Ferrier, A. Guisan, A. S. Hansen, D. W. Hilbert, C. Loehle, C. Margules, M. New, M. J. Sobel, D. R. B. Stockwell. Forecasting the Effects of Global Warming on Biodiversity. BioScience. Vol. 57, Iss.3, 2007.

[15] S. Salcedo-Sanz, Á. M. Pérez-Bellido, E. G. Ortiz-García, A. Portilla - Figueras, L. Prieto, F. Correoso. Accurate short-term wind speed prediction by exploiting diversity in input data using banks of artificial neural networks. Neurocomputing. Vol. 72, Iss. 4-6, 2009.

[16] M. Kanakidou, J. H. Seinfeld, S. N. Pandis, I. Barnes, F. J. Dentener, M. C. Facchini, R. Van Dingenen, B. Ervens, A Nenes, C. J. Nielsen, E. Swietlicki, J. P. Putaud, Y. Balkanski, S. Fuzzi, J. Horth, G. K. Moortgat, R. Winterhalter, C. E. L. Myhre, K. Tsigaridis, E. Vignati, E. G. Stephanou, and J. Wilson. Organic aerosol and global climate modelling: a review. Atmospheric Chemistry and Physics. Vol.5, Iss. 1053, 2005

[17] A. Xiu, J. E. Pleim. Development of a Land Surface Model. Part I: Application in a Mesoscale Meteorological Model.
Journal of Applied Meteorology and Climatology. Vol. 40, Iss. 2, 2001.

[18] J. E. Pleim. A Combined Local and Nonlocal Closure Model for the Atmospheric Boundary Layer. Part II: Application and Evaluation in a Mesoscale Meteorological Model. Journal of Applied Meteorology and Climatology. Vol.46, Iss. 9, 2007.

[19] B. Storm, J. Dudhia, S. Basu,A. Swift, I. Giammanco. Evaluation of the Weather Research and Forecasting model on forecasting low-level jets: implications for wind energy. Wind Energy. Vol.12, Iss. 1, 2009.

[20] W. C. Skamarock, J. B. Klemp. A time-split nonhydrostatic atmospheric model for weather research and forecasting applications. Journal of Computational Physics. Vol. 227, Iss. 7, 2008.

[21] R. Doherty, E. Denny, M. O'Malley. System operation with a significant wind power penetration. Power Systems Conference and Exposition. INSPEC Accession Number:8370473, 2004.

[22] T. M. Hamill, R. Hagedorn, J. S. Whitaker. Probabilistic Forecast Calibration Using ECMWF and GFS Ensemble Reforecasts. Part II: Precipitation. Monthly Weather Review. Vol.136, Iss. 7, 2008.

[23] W. Zheng,, H. Wei, Z. Wang, X. Zeng, J. Meng, M. Ek, K. Mitchell, J. Derber. Improvement of daytime land surface skin temperature over arid regions in the NCEP GFS model and its impact on satellite data assimilation. Journal of Geophysical Research. 117, D06117, doi:10.1029/2011JD015901, 2012. 